Carnets de géographes

GÉOGRAPHES.

$3 \mid 2011$

Les géographies des enfants et des jeunes

\title{
Les albums pour enfants et les géographies de l'enfance
}

L'exemple des représentations de la maison

Leïla Frouillou

\section{OpenEdition}

Journals

Édition électronique

URL : http://journals.openedition.org/cdg/2447

DOI : $10.4000 /$ cdg. 2447

ISSN : 2107-7266

Éditeur

UMR 245 - CESSMA

Référence électronique

Leilla Frouillou, « Les albums pour enfants et les géographies de l'enfance », Carnets de géographes [En ligne], 3 | 2011, mis en ligne le 01 décembre 2011, consulté le 23 septembre 2020. URL : http:// journals.openedition.org/cdg/2447 ; DOI : https://doi.org/10.4000/cdg.2447

Ce document a été généré automatiquement le 23 septembre 2020.

\section{c) (i) $९$}

La revue Carnets de géographes est mise à disposition selon les termes de la Licence Creative Commons Attribution - Pas d'Utilisation Commerciale - Pas de Modification 4.0 International. 


\title{
Les albums pour enfants et les géographies de l'enfance
}

\author{
L'exemple des représentations de la maison
}

\section{Leïla Frouillou}

Je remercie vivement Elise Olmedo, Gaëlle Reneteaud et les relecteurs des Carnets pour leurs conseils et leurs remarques.

1 Amatrice d'albums pour enfants, c'est presque naturellement, et avec grand plaisir ${ }^{1}$, que j'ai « profité » d'un travail de recherche de master, sur l'apprentissage des normes socio-spatiales par les enfants à l'école maternelle, pour m'interroger sur les rapports entre littérature jeunesse et géographie. La place des livres dans l'espace scolaire était évidente (coin bibliothèque, lectures d'histoires par le professeur) mais je souhaitais compléter l'analyse des albums comme supports pédagogiques en m'intéressant à leur contenu. Ce texte met en évidence l'intérêt de la littérature jeunesse comme source pour les géographies de l'enfance, en développant l'exemple des représentations de la maison dans des albums destinés aux enfants de 3 à 6 ans $^{2}$. N'étant pas spécialiste de géographie littéraire ni de littérature jeunesse, il s'agit seulement ici de souligner comment de telles analyses peuvent compléter efficacement l'observation des pratiques enfantines. À travers les albums pour enfants, on peut appréhender la vision d'un adulte (l'auteur) sur les pratiques et représentations de l'espace des enfants, sachant que cette vision participe à la construction de ces représentations (les lecteurs étant les enfants). Après quelques considérations sur la géographie littéraire et la place qu'y occupe la littérature jeunesse, je reviendrai sur la définition des albums pour enfants comme objet d'étude, avant d'analyser les représentations d'espaces domestiques dans plusieurs d'entre eux, notamment dans quelques livres de Claude Ponti. Cet auteur a en effet marqué les lectures et les imaginaires de nombreux enfants des années 1990 (dont je fais partie). Il continue de publier à l'Ecole des Loisirs, et ses albums sont bien représentés dans les salles de classe de maternelle. Ce texte est donc une sorte d'essai, car comme le rappelle Henri Desbois : « S'intéresser en géographe à la littérature, c'est aussi imaginer pouvoir écrire autrement la géographie, quitte à s'écarter un peu des canons de l'écriture académique »(Desbois, $2002: 4$ ). 


\section{La littérature jeunesse : une source pour les géographies de l'enfance}

2 Franco Moretti met en évidence les liens entre géographie et littérature : "La géographie est un aspect essentiel du développement et de l'invention littéraires ; c'est une force active, concrète, qui imprime sa marque sur les textes, sur les intrigues, sur les types d'attente» (Moretti, 2000: 9). De quelle façon le texte littéraire peut-il participer à l'analyse géographique ? Les recherches sur la géographie et la littérature tiennent une place réduite au sein de la géographie française (Chevalier, 2001). On peut citer néanmoins les travaux d'Armand Frémont, Paul Claval, Antoine Bailly et JeanLouis Tissier. Selon Michel Chevalier (2001), on peut répartir les recherches sur le sujet en deux groupes: d'une part celles qui tentent d'expliquer par des facteurs géographiques la formation et la personnalité des écrivains, la signification de leurs œuvres, les caractéristiques littéraires des villes et des régions, « dans la postérité de Taine », et de l'autre, l'approche par l'espace vécu, prépondérante aujourd'hui : «on désire beaucoup moins expliquer par la géographie des faits littéraires que demander à la littérature une meilleure compréhension du monde et des hommes " (Chevalier, 2001 : 15). C'est cette deuxième approche qui est privilégiée ici : quel éclairage la littérature jeunesse peut-elle apporter sur les pratiques des enfants et leurs représentations socio-spatiales?

3 La question posée par Jen-Louis Tissier : « Y a-t-il des genres plus propices que d'autres pour traduire, exprimer, des questionnements ou des expériences géographiques?» (2007 : 244), invite à s'interroger sur la place de la littérature jeunesse. Cette dernière ne semble pas être une référence pour les géographes, qui s'intéressent souvent à une littérature classique plus « sérieuse » que cette "paralittérature » (Clémentine, 2011). Ce manque d'intérêt des géographes peut renvoyer aux raisons avancées par Jean-Louis Tissier à propos de la poésie : «La singularité du texte poétique, la distance qu'il prend avec le référent, la "réalité", le travail de l'auteur sur le langage, la disparition du registre descriptif, expliquent sans doute les réticences des géographes» (1992: 247). Or, la littérature jeunesse éveille notre intérêt géographique par sa puissance d'évocation, et le rôle qu'y jouent l'image, et plus largement l'imaginaire. On pourra ainsi s'appuyer sur l'analyse de l'image poétique de Gaston Bachelard, dont les enquêtes "visent à déterminer la valeur humaine des espaces de possession, des espaces défendus contre des forces adverses, des espaces aimés » $(1957: 17)$, autrement dit des espaces vécus. Il s'intéresse en particulier à la maison dans cette exploration des espaces de l'intime, et ses réflexions sur ce thème, comme sur le nid, la coquille ou les coins, sont essentielles pour comprendre le pouvoir d'évocation des albums pour enfants étudiés ici.

4 Ces livres sont utiles pour comprendre l'« expérience humaine de l'espace, le sens des lieux » dont parle Philippe Gervais-Lambony (2007 : 276). La littérature jeunesse est une fenêtre sur les représentations des pratiques spatiales enfantines (lecteurs ciblés) par des adultes (les auteurs). Elle nous renseigne donc de façon générale sur les comportements enfantins attendus dans un contexte social et culturel spécifique. L'étude des albums jeunesse permet ainsi d'analyser une vision d'adulte sur les pratiques et représentations spatiales des enfants. Comme le souligne Anne Dupin (2004) : 
«L'univers pontien, avec ses multiples références culturelles et artistiques, ses thèmes et son langage propre aux territoires de l'enfance, son originalité dans les formes, les couleurs, la typographie ou le découpage scénique, manifeste des propositions narratives qui impliquent fortement l'émotionnel de l'enfant et coïncident avec son horizon d'attente. "

Cette vision adulte ne s'oppose donc pas aux attentes des lecteurs, aux pratiques et aux représentations enfantines effectives.

\section{Un objet d'étude à définir : les albums pour enfants}

On peut étudier la littérature dans l'espace ou l'espace dans la littérature (Moretti, 2000). La première approche invite à souligner la place et le statut de l'objet « album » dans les pratiques enfantines, défini dans les dictionnaires comme un livre, souvent de grand format, dans lequel l'illustration ou les images dominent. Ces albums sont présents dans plusieurs espaces fréquentés par les enfants : à l'école, dans les centres de loisirs ou parfois chez eux. Cet objet fait partie de leur quotidien. L'observation menée dans deux écoles maternelles montre un attachement aux livres: les enfants apprécient la lecture d'histoires par le professeur et peuvent consulter les albums dans le coin bibliothèque (Frouillou, 2011). Assis sur des poufs ou de petites banquettes, ils choisissent avec soin des livres qu'ils connaissent pour les avoir vus/lus plusieurs fois, ils en observent les images et les commentent. La lecture de ces livres s'appuie sur les dessins et l'imagination des enfants peut se combiner au souvenir de l'histoire ${ }^{3}$.

7 J'analyse ici quelques représentations d'espaces domestiques dans ces albums, autrement dit d'adopter la deuxième approche définie par Franco Moretti : l'espace dans la littérature. Mais comment mener une telle analyse? Comme pour toutes les sources littéraires, on doit s'interroger sur la valeur documentaire des albums jeunesse. Les œuvres littéraires sont le lieu de distorsions de la réalité qui dépendent en partie du public visé (Chevalier, 2001). Les albums jeunesse jouent explicitement avec la réalité et la détournent pour stimuler l'imaginaire des enfants. On peut s'interroger sur les intentions des auteurs d'albums pour enfants : quelles réactions cherchent-ils à susciter chez leurs lecteurs? La dimension ludique est particulièrement visible dans les albums de Claude Ponti, ce que confirme le petit livre de Lucie Cauwe sur cet auteur (2006). La valeur documentaire des albums jeunesse n'a donc de sens que dans le cadre d'une approche par les représentations et les codes sociaux.

8 D'autre part, l'analyse géographique du contenu d'albums jeunesse implique de s'attacher aussi bien à l'histoire (qui comprend le plus souvent une morale, des représentations sociales attachées aux personnages, aux situations et aux lieux), qu'aux dessins qui orientent la lecture. Ici, ce n'est pas tant l'aspect documentaire des dessins que leur puissance d'évocation qui semble essentielle. G. Bachelard souligne ainsi que le dessin peut déclencher la rêverie, en particulier lorsqu'il représente un espace domestique :

«Ces anciennes maisons, nous pouvons les dessiner, en donner par conséquent une représentation qui a tous les caractères d'une copie du réel [...] Mais cette représentation extérioriste, si seulement elle manifeste un art du dessin, un talent de représentation, la voici qui se fait insistante, invitante et que le seul jugement du bien rendu, du bien fait, se continue en contemplation et en rêverie. La rêverie revient habiter le dessin exact. La représentation d'une maison ne laisse pas longtemps un rêveur indifférent » (1957: 59-60) 
On peut penser que cela est d'autant plus vrai que le dessin ne se veut pas une fidèle représentation de l'espace et cherche à stimuler l'imagination enfantine.

Enfin, il faut rappeler que la problématique oriente la lecture : "Dans tous les cas, la lecture est intéressée : l'enseignant, le chercheur, applique à l'œuvre une attention sélective et finalisée, une grille qui exclut, explicitement ou non, le critère littéraire " (Tissier, 1992 : 236). Cela renvoie au " hiatus », décrit par Bertrand Levy (1997), entre la démarche de l'auteur qui représente le monde comme un tout foisonnant et le scientifique qui le découpe pour le comprendre. Il s'agit ici d'appréhender la représentation de l'espace domestique et de ses normes qui est donnée à lire aux enfants, et qui peut donc contribuer à régler leurs comportements comme l'encadrement pédagogique ou familial qui s'appuie en partie sur une organisation fonctionnelle de l'espace (Frouillou, 2011). L'objet d'étude est donc, comme l'explique Franco Moretti, "dans une large mesure imaginaire " (2000:9). Je pense que cette interrogation sur les espaces domestiques découle au moins en partie de mon propre rapport aux albums, qui renvoient à la sphère de l'intimité (lectures en famille avant d'aller se coucher). Mon attachement à ces objets a orienté ma lecture "géographique ", centrée sur les représentations des espaces de la vie quotidienne, et en particulier de l'espace domestique, qu'ils donnent à voir et à lire. Ces représentations prennent le plus souvent la forme de la «maison» et s'appuient sur des codes sociaux et culturels que je vais maintenant analyser.

\section{Maisons archétypes ou atypiques : jeux autour des codes des espaces domestiques}

11 On peut considérer que les albums jeunesse sont porteurs de codes sociaux et spatiaux, mis en récit et en image par des adultes (les auteurs), pour des enfants. Les représentations de la maison sont très présentes dans les livres pour enfants, et on pourrait reprendre la remarque de Bachelard (1957:23): "Une sorte d'attraction d'images concentre les images autour de la maison ». Cela renvoie à la place de l'espace domestique dans l'imaginaire spatial des enfants: il est le premier espace connu et approprié par les enfants. Cet espace de référence est donc comme le souligne JeanFrançois Staszak (2001: 346) : «l'espace le plus approprié, le plus chargé, celui qui porte le plus d'émotions et d'affects, de souvenirs et d'espoirs. Le lien entre identité et territoire explique que l'espace domestique participe de la "conscience individuelle" (Tuan, 1982). Associé à la sphère privée, à la famille et au corps, cet "espace physique" concourt à la composition du "territoire d'intimité" et donc à la construction du soi, selon le psychanalyste R. Neuburger ». Cela est d'autant plus vrai pour les enfants que : «De tous les espaces fréquentés par l'enfant, celui de la maison est sans doute le premier à découvrir, le plus familier, le plus familial, le plus influent dans la construction de ses repères spatiaux et affectifs » (Le Run, 2006 : 27). Les géographies de l'enfance s'intéressent le plus souvent aux espaces fréquentés quotidiennement par les enfants, en premier lieu les espaces domestiques et scolaires. La maison comme entité occupe donc une place importante dans l'imaginaire spatial et affectif des enfants. Jean-Louis Le Run revient ainsi sur l'importance de cet espace intime, ce refuge rassurant qui renvoie à une dialectique de l'intérieur/extérieur :

«La maison est un abri, elle est ce corps enveloppant et protecteur qui vient redoubler, de l'extérieur, l'enveloppe maternelle. Entre les murs extérieurs et 
l'enveloppe corporelle s'étend l'espace de la maison. Ni dedans de soi, ni dehors, c'est un lieu intermédiaire. C'est l'espace de l'intimité familiale. Il est plus ou moins étendu selon les moyens et l'histoire de la famille, plus ou moins sophistiqué, et son aménagement varie selon les cultures. Il est ce chez-soi familier que, simultanément, nous pouvons fermer ou ouvrir à d'autres que nous laissons entrer, pour partager notre intimité à des degrés divers et généralement assez maîtrisés. La maison cultive ce paradoxe d'un dedans-dehors, sorte d'extériorisation de notre moi, évagination qui simultanément nous entoure » (2006:28).

Les contes pour enfants comportent de nombreuses représentations de maisons, plus ou moins archétypales selon les éditions. Le Petit Chaperon Rouge en est un bon exemple : la maison de la grand-mère peut ainsi être utilisée dans des exercices de graphisme en classe (fenêtres symétriques par rapport à une porte, briques, fumée qui s'échappe de la cheminée, toit pointu), ou bien dans des exercices de reconnaissance de mots ( "maison »). De façon générale, l'image archétypale de la maison est très présente à l'école maternelle: outre les exercices, on la retrouve sous forme de panneau (le déplacement de l'étiquette portant le nom de chaque élève vers un autre panneau représentant l'école permettant de signifier le passage de l'espace domestique à l'espace scolaire), dans certains dessins des enfants qui sont affichés dans les classes ${ }^{4}$, et bien sûr dans les jouets.

13 Il m'a semblé intéressant de choisir des albums qui ne soient pas des contes, mais qui restent susceptibles d'être connus par un assez grand nombre d'enfants, afin de mettre en évidence la diversité des approches de la littérature jeunesse 3-6 ans. Les représentations des espaces domestiques et en particulier de maisons sont très variées selon le thème et le ton de l'album, c'est-à-dire l'imaginaire auquel fait appel l'auteur. On peut dans un premier temps distinguer deux catégories de représentations de ces espaces domestiques: celles qui les mettent en scène de façon classique, et celles qui déforment les normes habituelles de ces espaces. Par exemple, l'Encyclopédie Babar Larousse, Babar à la maison présente chaque espace fonctionnel de la maison de façon très « classique » (les salles d'eau, les chambres, le grenier, le salon, la salle à manger, les espaces extérieurs) avec le mobilier correspondant et les actions qui s'y déroulent.

Mais, les livres pour enfants jouent toujours avec les normes socio-spatiales, de façon plus ou moins appuyée. Cela peut être ténu: dans Babar à la maison, c'est la forme générale de la maison qui est étrange tout en s'inscrivant dans le schéma type maisongarage-cabane au fond du jardin. Pélagie la sorcière constitue un exemple intéressant de représentation de l'espace domestique et des normes sociales qui y sont associées. On retrouve l'image de la maison comme être vertical soulignée par Gaston Bachelard, « la verticalité est assurée par la polarité de la cave et du grenier » $(1957: 35)$, à travers les nombreuses tours de la demeure (Figure $n^{\circ} 1$ ). D'une maison à la fois très classique (forme, fonctionnalité, aménagement des pièces) et originale (complètement noire, tours et escaliers, placard aux balais avec un chaudron), la sorcière passe à un espace domestique archétypal, où les seules pièces visibles sont la salle de bain, la chambre et le salon. La couleur noire représente ainsi une rupture par rapport aux codes de la maison stéréotypée aux murs clairs et au toit en pente rouge que l'on retrouve dans l'exemple de Babar, mais aussi dans des représentations moins archétypales comme les maisons d'Hippolène et de Zouk (Figures $\mathrm{n}^{\circ} 4$ et 5 ). De façon générale, les couleurs utilisées par les auteurs témoignent des normes sociales dans les représentations de la maison: l'espace domestique tortueux d'okilélé, décrit plus bas, est sombre, comme 
celui de Pélagie. La transformation de sa maison symbolise ainsi la normalisation de Pélagie, qui lui permet de retrouver son chat noir Rodolphe.

Figure $n^{\circ} 1$ : Couleurs et espaces domestiques, l'exemple de la maison de Pélagie

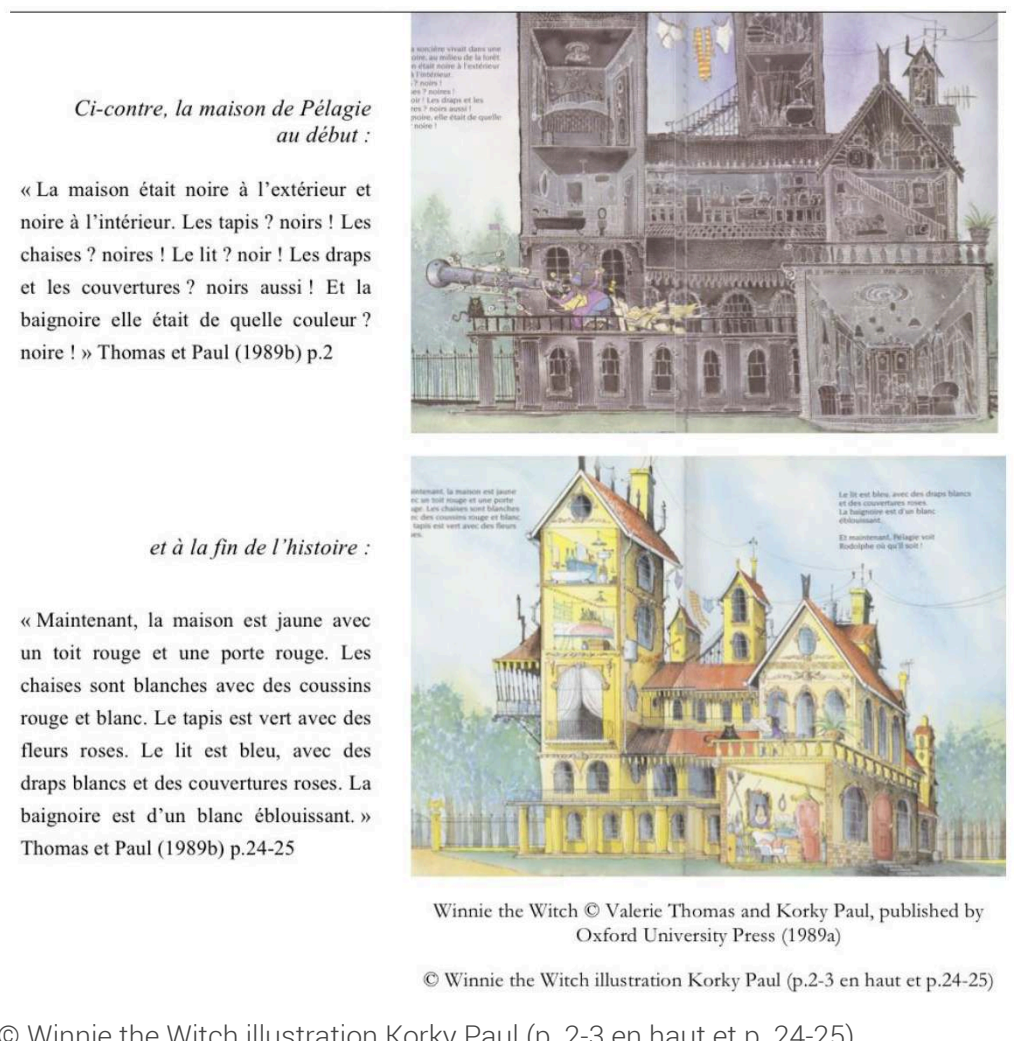

Tout en respectant les codes (la maison en couleur est une fin heureuse dans Pélagie la sorcière), ces livres les discutent, à la marge et souvent avec humour. Cela ne constitue pas un trait spécifique de la littérature jeunesse, car comme le souligne Jean-Louis Tissier :

«L'écriture créative dépayse et déterritorialise, elle oblige le lecteur à faire l'expérience d'un monde nouveau, à mettre en question les certitudes et les causalités du monde ancien. La littérature dérange, introduit du jeu dans les représentations habituelles [...] Ces effets sont d'autant plus efficaces que l'œuvre est vraiment contemporaine » $(1992: 253)$.

On peut également s'appuyer sur l'analyse que fait Gilles Brougère (1989) des représentations de l'habitat dans les jouets. Il montre que l'image de la maison, renvoyant à des valeurs de sécurité, de confort, d'intimité, et d'unité familiale, est en partie représentée dans les jouets par le toit à double pente, qui suffit à "évoquer la maison comme lieu clos» (1989: 15). Les différentes catégories de jouets (premier âge, maisons univers, univers mondes, construction, maisons à la taille de l'enfant, etc.) utilisent avant tout le stéréotype de la maison classique. Mais cela peut aller de l'élément le plus réaliste (puzzle par exemple) à une allusion discrète (souvent liée au toit à double pente). La maison doit rapidement être identifiée par l'enfant, ce qui explique la place des archétypes :

«Le jouet se complaît dans l'évocation, dans l'indice à partir duquel le joueur développe son jeu. Pour ce faire, il favorise les représentations archétypales, les clichés, les stéréotypes qui s'adressent à tous, qui sont supports de significations 
avant d'être représentatifs d'une réalité quelconque. [...] Moins qu'une image de notre monde le jouet nous propose une image de nos mythes, de notre façon de construire l'enfance. L'habitat, comme tout le reste, n'est pas présent dans le jouet en fonction de ce qui est, mais à travers la médiation des images qui structurent nos différentes représentations de l'enfant, entendu comme représentation de l'enfant par l'adulte mais aussi représentation du monde extérieur par l'enfant lui-même » (1989: 31).

Cette analyse s'applique également aux albums pour enfants étudiés ici : les images de la maison, qui s'appuient en partie sur des représentations archétypales, sont à la fois révélatrices de la vision d'un adulte de l'enfance, et en un sens de la façon dont les enfants se représentent "effectivement» le monde. Les albums pour enfants participent, parmi d'autres représentations, discours et pratiques, à la construction des représentations enfantines, qui s'appuient sur des codes sociaux et spatiaux.

Néanmoins, certains livres pour enfants remettent très clairement en cause les stéréotypes de l'espace domestique. Ils en jouent pour faire rire et construire des mondes étranges. Dans les albums de Claude Ponti, on trouve des maisons biscornues, tortueuses, pleines d'escaliers et de recoins. Okilélé, rejeté par sa famille, se construit une maison souterraine à partir du "placard de sous l'évier » de la maison familiale (Figure $\left.\mathrm{n}^{\circ} 2\right)$. Ce coin est déjà en lui-même une référence à l'espace domestique :

"Tout coin dans une maison, toute encoignure dans une chambre, tout espace réduit où l'on aime à se blottir, à se ramasser sur soi-même, est, pour l'imagination une solitude, c'est-à-dire le germe d'une chambre, le germe d'une maison" (Bachelard, 1957 : 130).

19 Les coins facilitent l'appropriation de l'espace par les enfants : les salles de classes des écoles maternelles s'organisent en coins (coin jeux, coin bibliothèque, coin construction, etc.) pour faciliter le repérage des enfants, condition nécessaire d'une certaine autonomie scolaire. Les coins renvoient donc bien à la sphère de l'intime. Dans Okilélé, un espace domestique se construit effectivement à partir du coin, du " placard de sous l'évier ». Les espaces fonctionnels sont originaux : exceptée la salle de lecture, on trouve des jardins intérieurs en terrasses, une forêt de champignons, une piscine. Les salles sont reliées par des échelles et des cordes. Cette maison souterraine, appelée «Maison-Sous-la-Terre " (Ponti, 1993: 16), rappelle les images de l' "ultra-cave» décrites par Gaston Bachelard, comme la demeure du rêveur qui :

«veut les souterrains des châteaux forts de la légende où de mystérieux chemins faisaient communiquer par-dessous toute enceinte, tout rempart, tout fossé, le centre du château avec la forêt lointaine. Le château planté sur la colline avait des racines fasciculées de souterrains. Quelle puissance pour une simple maison d'être bâtie sur une touffe de souterrains!» (1957 : 37).

Cela renvoie à la maison comme refuge, face aux brimades et aux moqueries de la famille d'Okilélé (les "rêves de huttes » décrits par Bachelard). La maison tortueuse peut également se lire comme une coquille, construite par Okilélé et son compagnon Martin Réveil, à la manière de la rêverie de Bernard Palissy ${ }^{5}$ : "Ils creusèrent de nombreux tunnels. Tous les matins, ils inventaient une pièce qui s'ajoutait aux autres " (Ponti, 1993: 16). Cette coquille prépare sa sortie, le début d'une aventure qui le mènera jusqu'à des planètes lointaines : « L'être qui se cache, l'être qui "rentre dans sa coquille" prépare une "sortie" » (Bachelard, $1957: 110)$. 
Figure $\mathrm{n}^{\circ} 2$ : La maison d'Okilélé, un refuge souterrain

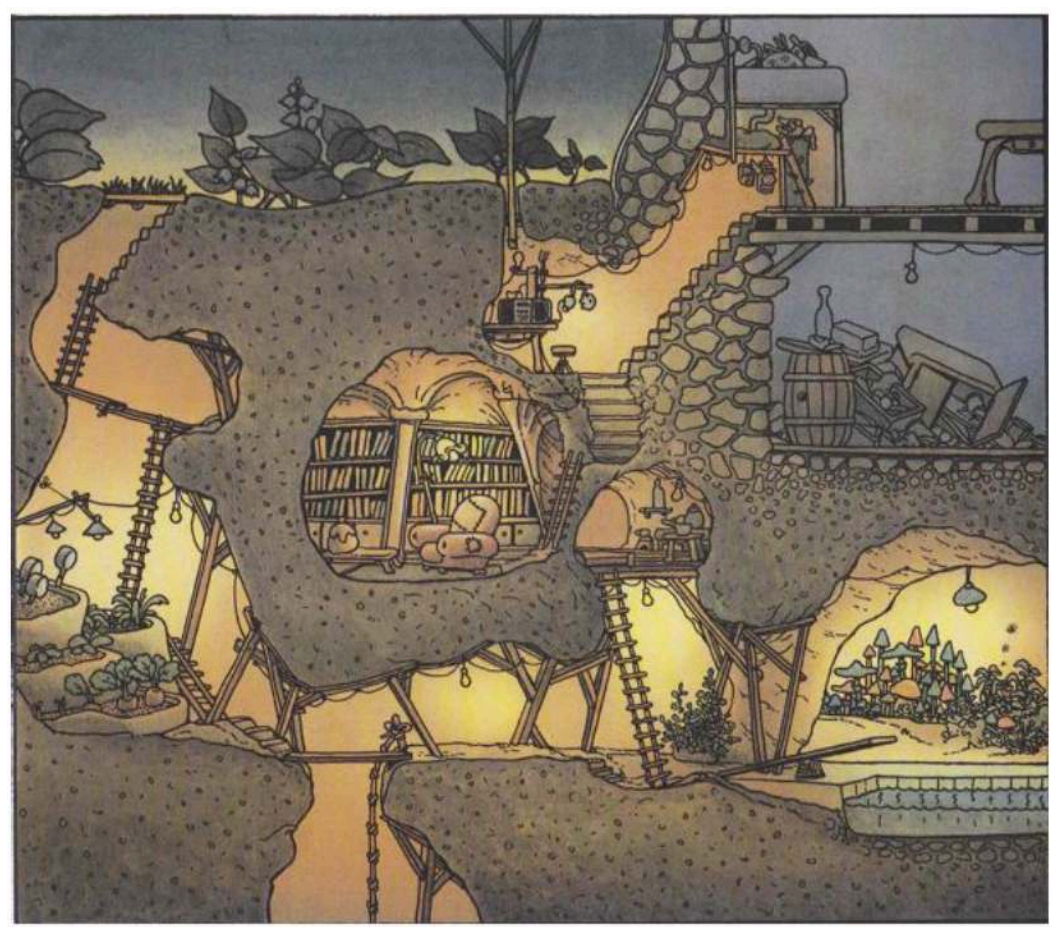

Ponti (1993) Okilélé, l'Ecole des loisirs, p.16

Ponti (1993) Okilélé, l'Ecole des loisirs p. 16

21 On retrouve des espaces domestiques encombrés, tortueux, pleins de contradictions et de détournements des fonctionnalités habituelles, dans d'autres albums de Ponti comme Le chien invisible, Le doudou méchant ou bien dans Parci et Parla. Dans ce dernier album, le frère et la sœur commencent par ranger la maison, sans pour autant la normaliser tout à fait (le lave-linge et la machine à laver ont toujours des pattes, figure $\left.n^{\circ} 3\right)$.

Figure $n^{\circ} 3$ : La maison de Parci et Parla, rangée sans être pour autant normalisée

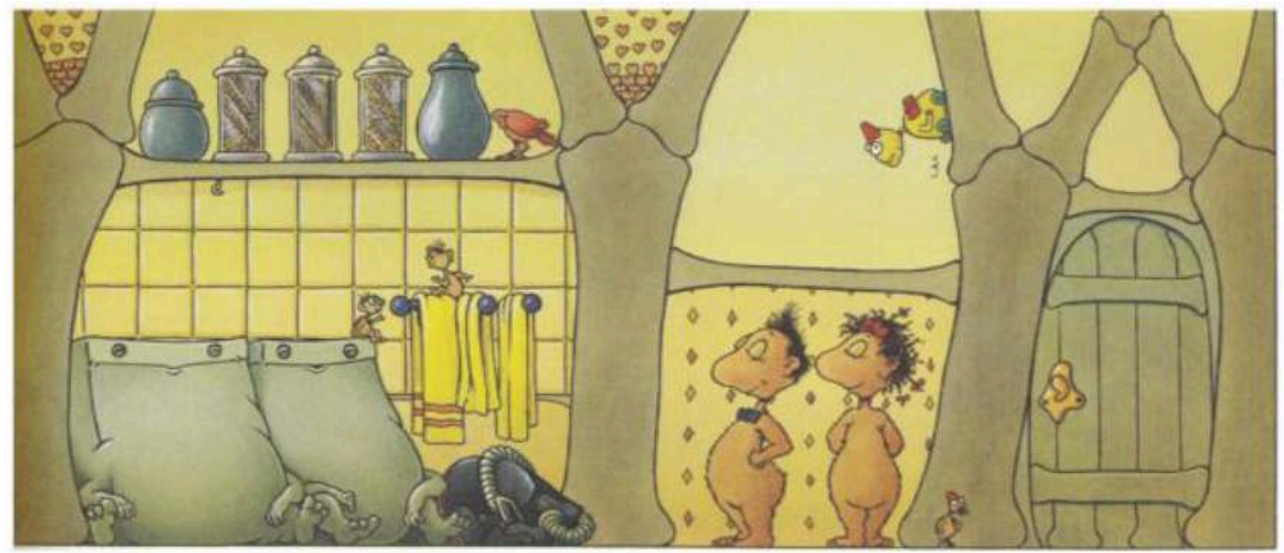

Ponti (1994) Parci et Parla, l'Ecole des loisirs, p.13

Ponti (1994) Parci et Parla, l'Ecole des loisirs, p. 13 
L'espace domestique d'Hippolène (figure $\mathrm{n}^{\circ} 4$ ) détourne lui aussi les codes de la maison. Intégrée à un arbre, cette maison comprend plusieurs tours dont la forme rappelle des fleurs, mais elle est tout de même pourvue d'une porte, de fenêtres, et d'un toit rouge. Certaines de ses ouvertures sont éclairées, ce qui rappelle une fois de plus l'analyse de Gaston Bachelard : «Par sa seule lumière, la maison est humaine. Elle voit comme un homme. Elle est un œil ouvert sur la nuit » (1957 : 48). L'espace domestique peut même devenir un personnage à part entière comme la "Mâzon» dans Le Nakakoué, ou la maison de la grand-mère du Chaperon Rouge croisée par Parci et Parla (Figure $n^{\circ} 5$ ). Cette maison est devenue aveugle au même titre que le loup ou la petite fille (car le livre n'a pas été ouvert depuis très longtemps, et il y fait noir)! On peut à nouveau citer Gaston Bachelard: "Ainsi, en face de l'hostilité, aux formes animales de la tempête et de l'ouragan, les valeurs de protection et de résistance de la maison sont transposées en valeurs humaines. La maison prend les énergies physiques et morales d'un corps humain » (1957 : 57). La Mâzon du Nakakoué n'est donc pas un cadre ou un décor mais un personnage qui renvoie au rôle que peut avoir la maison pour les lecteurs : un refuge, un lieu de l'intime séparé du "dehors" menaçant où se produisent les aventures des personnages de ces albums. Cet anthropomorphisme se retrouve dans les dessins des enfants comme le souligne Jean-Louis Le Run :

« Les enfants partagent avec les créateurs la tendance à faire de la maison, un être vivant. Le cinéma, en particulier américain, est plein d'exemples où la maison est un personnage à part entière sinon le personnage principal: maison heureuse, maison hantée, maison qui se transforme, qui devient menaçante, espace inanimé qui se met à vivre sa propre vie. [...] Lorsqu'on demande aux jeunes enfants de dessiner une maison, ils produisent souvent le même modèle, avec deux fenêtres, une porte, une cheminée, une allée et un toit pointu: modèle appris ou fantasme partagé ? Peut-être les deux, le stéréotype relevant sans doute autant des images trouvées dans les livres d'enfant, des modèles appris des parents ou des enseignants que de l'anthropomorphisme inhérent à cette représentation. Cette maison reprend l'essentiel d'un visage ou d'un corps, homologie relevée par les psychanalystes et les psychologues. » $(2006: 32)$ 
Figure $n^{\circ} 4$ : La maison d'Hippolène

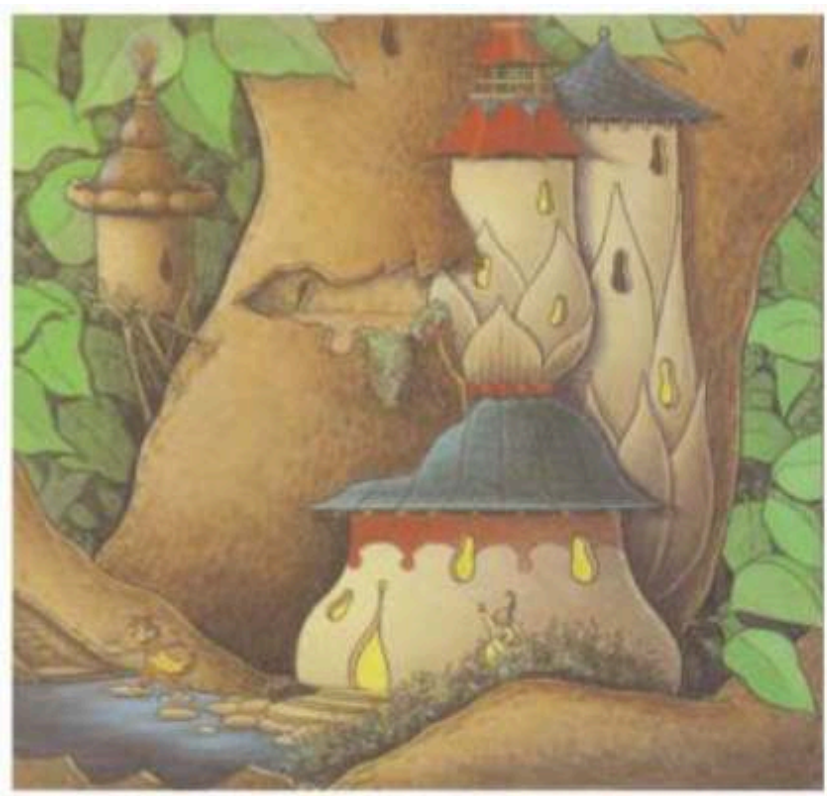

\section{Ponti (1992) L'arbre sans fin, l'Ecole des loisirs, p.43}

Figure $n^{\circ} 5$ : Des maisons qui deviennent des personnages à part entière

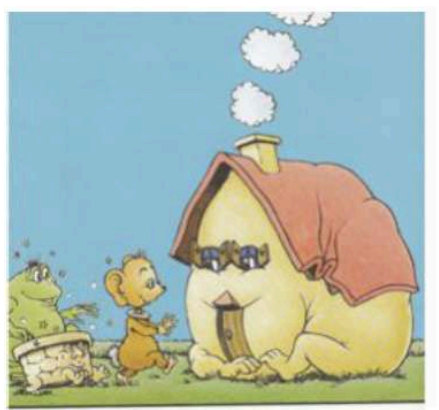

Ci-contre, la Mâzon et Zouc des loisirs, p.42 :

" "Quand on sera à Rivé, on sera chez moi", dit Zouc, "et on habitera tous ensemble." "Moi je serai ta Mâzon quand tu seras grand", dit la Mâzon. »

Ci-dessous, la maison aveugle de la grand-mère du Chaperon

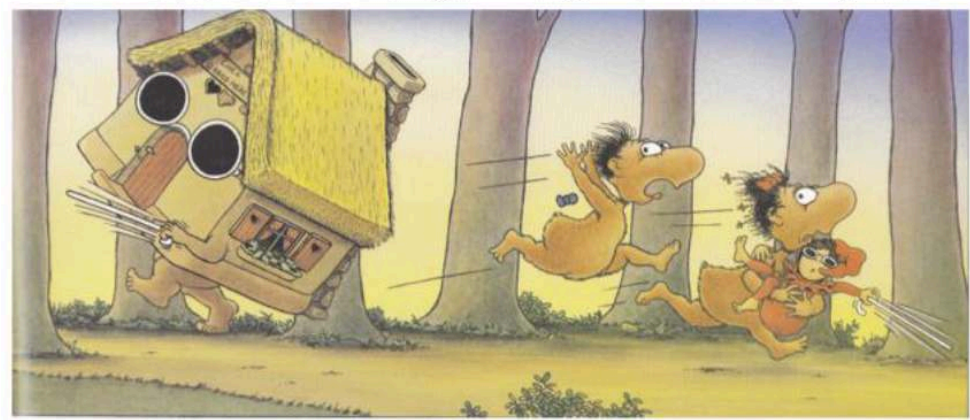

Ponti (1994) Parci et Parla, l'Ecole des loisirs, p.23

En haut : Ponti (1997) Le Nakakoué, l'Ecole des loisirs, p. 42

En bas : Ponti (1994) Parci et Parla, I'Ecole des loisirs, p. 23 

jouent un rôle de repère pour les personnages, en symbolisant souvent le but ou la fin de l'aventure. Les maisons sont également par extension autant de repères pour les enfants, qui s'identifient aux personnages de l'histoire. Les auteurs s'appuient donc sur les sentiments que peuvent éprouver les enfants par rapport à ce lieu intime pour structurer leur histoire, et faciliter l'investissement émotionnel des lecteurs. Par exemple, Parci et Parla reviennent chez eux à la fin de la journée et retrouvent leurs parents. La "Mâzon » du Nakakoué, personnage à part entière, pourvue de pattes et de fenêtres qui sont autant d'yeux, symbolise également la normalisation et la fin de l'histoire (Figure $\mathrm{n}^{\circ} 5$ ). Comme le souligne Bachelard, ces espaces domestiques renvoient à la sphère de l'intime : « la transposition à l'humain se fait tout de suite, dès qu'on prend la maison comme espace de réconfort et d'intimité, comme un espace qui doit condenser et défendre l'intimité » (1957: 59). Cela se traduit également dans ces espaces domestiques par la présence familiale (Babar et toute la famille, Pélagie et Rodolphe, le retour d'Okilélé dans la maison familiale, les parents de Parci et Parla). fonctionnalité des espaces domestiques, donc à des détournements. Les albums offrent ainsi aux enfants des espaces domestiques "alternatifs", où un certain nombre de codes architecturaux et fonctionnels sont inversés voire éliminés. Mais le fait de jouer avec les stéréotypes souligne en filigrane la connaissance qu'en ont les enfants: si la maison d'okilélé les fait rire, rêver ou imaginer, c'est qu'ils sont bien conscients de la transgression des normes socio-spatiales caractéristiques de leur propre espace domestique, et de la plupart des espaces qu'ils fréquentent quotidiennement (comme l'école, Frouillou, 2011).

De façon générale, les albums permettent de souligner le lien entre la manipulation de l'espace par les enfants (déplacements et marquages) et l'imaginaire qui la sous-tend, comme le montre par exemple La chaise bleue de Claude Boujon (1996). Les deux personnages de cet album, Escarbille et Chaboulo, trouvent une chaise dans le désert et explorent toutes les possibilités qu'elle offre en termes de jeux (Figure $n^{\circ} 6$ ). Ce livre illustre le lien entre manipulation du mobilier et imagination, et montre la dimension ludique de la transgression des normes spatiales, de la transformation de l'espace. Il permet d'appréhender une autre vision de ces objets et de ces lieux, certes adultocentrée mais qui tente de rendre compte de certaines pratiques enfantines. Dans cet album, ces manipulations, ces transgressions restent néanmoins ponctuelles car les adultes sont là pour faire respecter les codes. A la fin du livre, un dromadaire s'assoit sur la chaise et interrompt les jeux d'Escarbille et Chaboulo (Figure $n^{\circ} 6$ ). Cette analyse complète l'observation des processus de socialisation des enfants par l'espace à l'école maternelle, qui s'appuient sur deux logiques complémentaires: d'une part un encadrement pédagogique des comportements enfantins, et d'autre part des transgressions qui permettent une appropriation de l'espace (Frouillou, 2011). 
Figure $n^{\circ} 6$ : Imaginaire et normes socio-spatiales, l'exemple d'Escarbille et Chaboulo

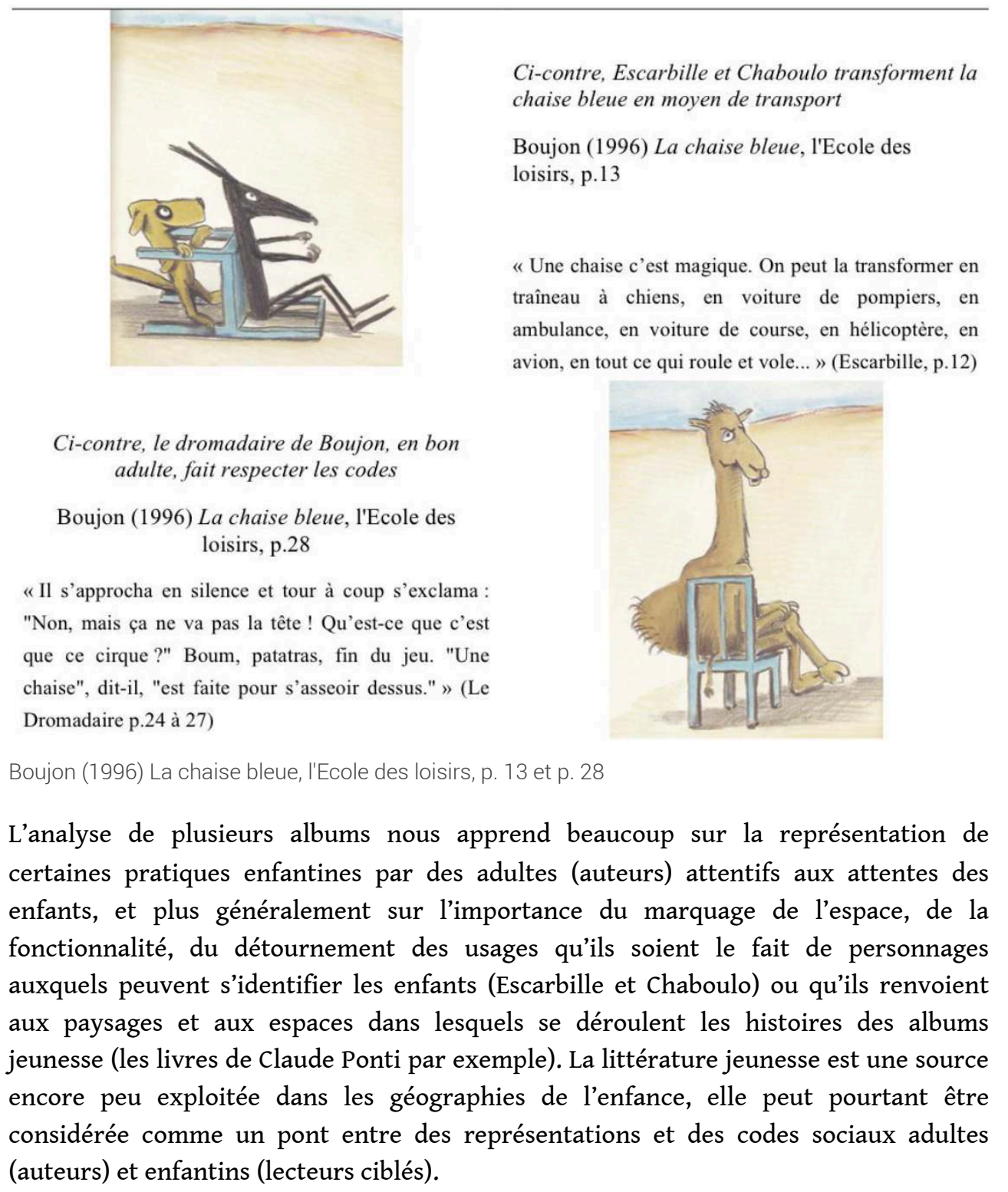

Ci-contre, Escarbille et Chaboulo transforment la

Boujon (1996) La chaise bleue, l'Ecole des

oisirs, p.13

« Une chaise c'est magique. On peut la transformer en à chiens, en voiture de pompiers, en mbulance, en voiture de course, en hélicoptère, en ion, en tout ce qui roule et vole... » (Escarbille, p.12)

i-contre, le dromadaire de Boujon, en bon ulte, fait respecter les codes

$$
\text { loisirs, p.28 }
$$

«Il s'approcha en silence et tour à coup s'exclama : "Non, mais ça ne va pas la tête! Qu'est-ce que c'est que ce cirque ?" Boum, patatras, fin du jeu. "Une chaise", dit-il, "est faite pour s'asseoir dessus." » (Le Dromadaire p.24 à 27

L'analyse de plusieurs albums nous apprend beaucoup sur la représentation de certaines pratiques enfantines par des adultes (auteurs) attentifs aux attentes des enfants, et plus généralement sur l'importance du marquage de l'espace, de la fonctionnalité, du détournement des usages qu'ils soient le fait de personnages auxquels peuvent s'identifier les enfants (Escarbille et Chaboulo) ou qu'ils renvoient aux paysages et aux espaces dans lesquels se déroulent les histoires des albums jeunesse (les livres de Claude Ponti par exemple). La littérature jeunesse est une source encore peu exploitée dans les géographies de l'enfance, elle peut pourtant être (auteurs) et enfantins (lecteurs ciblés).

\section{BIBLIOGRAPHIE}

BACHELARD G. (1957, réed. 2010) Poétique de l'espace, Paris, PUF, 215 p.

BROUGERE G. (1989) La représentation de l'habitat dans le jouet, Paris, Comité pour le développement de l'espace pour le jeu, $31 \mathrm{p}$.

CAUWE L. (2006) Ponti Foulbazar, L'École des Loisirs, Paris.

CHEVALIER M. (2001) Géographie et Littérature, Paris, Société de Géographie, 260p. 
CLEMENTINE B. (2011) « Ode à la paralittérature », http://clementinebleue.blogspot.com/ 2011/10/ode-la-paralitterature.html

DESBOIS H. (2002) « Introduction : Territoires littéraires et écriture géographique », Géographie et Cultures, $\mathrm{n}^{\circ} 44$, pp. 3-4

DUPIN A. (2004), « Activités autour de l'œuvre de Claude Ponti », site CRDP Académie de CréteilScérén, consulté le 22/11/2011 :

http://www.crdp.ac-creteil.fr/telemaque/document/ponti01.htm

FROUILLOU L. (2011) « Géographie d'un espace conçu pour les élèves, approprié par les enfants : L'école maternelle française », Carnets de Géographes, nํo 3.

GERVAIS-LAMBONY P. (2007) « Nouvel espace, nouvelle littérature, et retour. Ecrire les subusbs de Johannesburg. Remarques autour du recueil d'I. Vladislavic : The ExplodedView », Bulletin de l'Association des Géographes Français - Géographies, 2007/3, pp. 275-285

LEVY B. (1997) « Géographie culturelle, géographie humaniste et littérature. Position épistémologique et méthodologique ", Géographie et Cultures, $\mathrm{n}^{\circ} 21, \mathrm{pp} .27-44$

LE RUN J.-L. (2006) « L'enfant et l'espace de la maison », Enfances \& PSY, n 33, pp. 27-36.

MORETTI F. (2000) Atlas du roman européen : 1800-1900, Paris, Seuil, 235 p.

STASZAK J.-F. (2001) «L'espace domestique : pour une géographie de l'intérieur », Annales de géographie, vol. 110, $\mathrm{n}^{\circ}$ 620, pp. 339-363

TISSIER J.-L. (1992) « Géographie et littérature », dans Bailly A. et al.. (eds), Encyclopédie de géographie, Paris, Economica, pp. 235-255

TISSIER J.-L. (2007) « Géographie et littérature, présentation », Bulletin de l'Association des Géographes Français - Géographies, 2007/3, pp. 243-247

\section{Albums étudiés}

BOUJON C. (1996) La chaise bleue, Paris, l'École des Loisirs, 32 p.

Encyclopédie Babar Larousse (1990) Babar à la maison, Larousse, $44 \mathrm{p}$.

PONTI C. (1992) L'arbre sans fin, Paris, l'Ecole des loisirs, 48 p.

PONTI C. (1993) Okilélé, Paris, l'Ecole des loisirs, 48 p.

PONTI C. (1994) Parci et Parla, Paris, l'Ecole des loisirs, 48 p.

PONTI C. (1995) Le chien invisible, Paris, l'Ecole des loisirs, 40 p.

PONTI C. (1997) Le Nakakoué, Paris, l'Ecole des loisirs, 48 p.

PONTI C. (2000) Le doudou méchant, Paris, l'Ecole des loisirs, 56 p.

THOMAS V., PAULK., (1989a), Winnie the Witch, Oxford University Press, 24 p. et (1989b) Pélagie la sorcière, Toulouse, Milan, pour la version française.

\section{NOTES}

1. Comme l'explique Henri Desbois, c'est en partie « le côté ludique que peut revêtir l'étude de géographies de papier pour un géographe rassasié de réel » qui fonde cet intérêt des géographes pour la littérature $(2002: 3)$. 
2. Pour les lecteurs de la collection l'École des loisirs, il s'agit des sélections Titoumax (2 à 4 ans), Minimax ( 3 à 6 ans) et Kilimax (5 à 8 ans).

3. L'imbrication entre mémoire et imagination est soulignée par Gaston Bachelard : «Ainsi, en abordant les images de la maison avec le souci de ne pas rompre la solidarité de la mémoire et de l'imagination, nous pouvons espérer faire sentir toute l'élasticité psychologique d'une image qui nous émeut à des degrés de profondeur insoupçonnés » (1957:25).

4. Lors d'une observation de plusieurs semaines dans deux écoles maternelles (Frouillou, 2011), les enfants de grande section ont accepté volontiers de dessiner leur maison, mais ils ont refusé de dessiner leur école ou leur classe, jugée trop difficile. Ce manque d'intérêt pour l'espace scolaire, au profit de l'espace domestique, me semble renvoyer à la notion de «territoire de l'intimité » dont parle Jean-François Staszack (2001), cité plus haut.

5. Gaston Bachelard rappelle qu' « une rêverie de la construction par le dedans occupe Palissy plusieurs mois» (1957: 123). Le quatrième cabinet imaginé par Bernard Palissy «est une synthèse de la maison, de la coquille et de la grotte [...] Ce sera une grotte en forme de coquille enroulée [...] Par les bosses qui pendent, la demeure rocheuse reçoit le cauchemar de l'écrasement. Par la spirale qui s'enfonce dans la roche, elle reçoit une profondeur tourmentée » $(1957: 126)$.

INDEX

Thèmes : Carnets de lectures

\section{AUTEUR}

\section{LEİLA FROUILLOU}

Paris 1 Panthéon-Sorbonne

(UMR Géographie-cités - CRIA)

Géographie-Aménagement

leila.frouillou[at]gmail.com 